\section{AB1029 FIBRODYSPLASIA OSSIFICANS PROGRESSIVA: A CHALLENGE TO DIAGNOSE AND TO TREAT}

Mohammed Nashawi, Jan Henje Döring, Markus Bettendorf, Stefan Kölker, Georg Hoffmann, Andreas Ziegler. University Hospital Heidelberg, Center for Pediatrics and Adolescent Medicine, Heidelberg, Germany

Background: Fibrodysplasia ossificans progressiva (FOP) caused by mutations in the ACVR1 gene, which codes for activin receptor IA, a type I receptor of the bone morphogenetic protein (BMP) pathway. FOP is a very rare disease which usually begins in the first decade of life and characterized by congenital bilateral hallux valgus in combination with progressive heterotopic ossification in specific anatomical patterns preceded by inflammatory responses and soft tissue swelling.

Objectives: Our objective is to expand the differential diagnosis of recurrent swelling without trauma by presenting a case of Infant suffering from recurrent swellings and diagnosed to have FOP.

Methods: We used standard laboratory assessment and ultrasound to exclude other diseases. To confirm the diagnosis we used next generation sequencing to identify ACVR1 variants.

Results: A full-term male infant from non-consanguine parents delivered spontaneously after uncomplicated pregnancy. Initially bilateral hallux valgus, as well as limited motility in the metacarpophalangeal joint of D1 of the right hand was noted. At the age of 1.5 months, a severe swelling appeared on the left head without trauma. The further investigation showed no signs of retinal hemorrhage or intracranial injury. The swelling resolved spontaneously, but he readmitted to the hospital due to swellings of different localization in the head area. At the beginning a child abuse had been discussed with the family, although there was no concrete evidence.

At the age of 7 months he presented to our center with renewed swelling in the area of the forehead. Due to the characteristic symptom constellation, a genetic analysis was performed on ACVR1, which confirmed a de novo mutation c.617G> A, p.R206H

We started a combined therapy with Montelukast $4 \mathrm{mg}$ oral daily for specific inhibition of mast cell activation, Neridronat (Bisphosphonate) $2 \mathrm{mg} /$ $\mathrm{kg}$ as i.v. every 3 months for the modification of secondary ossification. As well as Prednisolone for 3 days $20 \mathrm{mg} / \mathrm{kg}$ i.v. in the flare up. At the beginning there were a significant reduction in the frequency and severity of new swellings, however after 9 Months he had frequent flare up with limitation of head movement. At that point we decided to start an off-label therapy with Imatinib. He responded well to the current therapy and the medication is well tolerated.

Conclusion: FOP is a very rare and important differential diagnosis of child abuse. With the characteristic symptom of congenital bilateral hallux valgus and unclear swellings, the diagnosis can be genetically secured. An early specific treatment concept for the avoidance of trauma as well as drug-based anti-inflammatory therapy are crucial for the clinical course and subsequent impairment of the patient. New targeted treatment approaches offer a promising option for the long-term improvement of disease progression and associated quality of life like Imatinib, which is a tyrosine kinase inhibitor used in the treatment of chronic myeloid leukemia in adult and children. A recent article showed that it decreased the flare up in 6 out of 7 patients with FOP.

\section{REFERENCES}

[1] Pignolo RJ, Shore EM, Kaplan FS. Fibrodysplasia ossificans progressiva: clinical and genetic aspects. Orphanet J Rare Dis. 2011;6:80.

[2] Hatsell SJ, Idone V, Wolken DM, Huang L, Kim HJ, Wang L, et al. ACVR1R206H receptor mutation causes fibrodysplasia ossificans progressiva by imparting responsiveness to activin A. Sci Transl Med. 2015;7 (303):303ra137.

[3] Luo Y, Alsamarah A, Zhang K, Hao J. Development of New Therapeutic Agents for Fibrodysplasia Ossificans Progressiva. Curr Mol Med. 2016;16 (1):4-11

[4] Kaplan FS, Andolina JR, Adamson PC, Teachey DT, Finklestein JZ, Ebb DH, Whitehead B, Jacobs B, Siegel DM, Keen R, Hsiao E, Pignolo RJ. Early clinical observations on the use of imatinib mesylate in FOP: A report of seven cases: Bone. 2018 Apr;109:276-280.

\section{AB1030 NEUROPSYCHIATRIC MANIFESTATIONS IN CHILDHOOD-ONSET SYSTEMIC LUPUS ERYTHEMATOSUS: RETROSPECTIVE STUDY IN ONE SINGLE CENTER}

Maria Kaleda, Irina Nikishina, Svetlana Glukhova. V. A. Nasonova Research Institute of Rheumatology, Pediatric, Moscow, Russian Federation

Background: Systemic lupus erythematosus is a complex autoimmune disorder characterized by multisystem involvement, including the nervous system, which elevate damage and mortality. The investigation of neuropsychiatric involvement in childhood-onset SLE (cSLE) is important in real clinical practice to improve the prognosis.

Objectives: The aim of this study was to assess neuropsychiatric manifestations (NM) in cSLE in one single center.

Methods: The study included all pts with cSLE who undergoing in-patient treatment in our center from 1992 to 2017 yy. Diagnosis of SLE was reviewed based on 2012 SLICC criteria. Data including sex, age at the time of onset, age at the time of diagnosis, physical examination, laboratory review, and neuropsychiatric inventory were extracted from our database. Classification of NM of cSLE was according to the 1999 American College of Rheumatology (ACR) neuropsychiatric manifestations of SLE case definitions.

Results: We included 218 consecutive pts with cSLE, of which 45 pts $(20.6 \%)$ had neuropsychiatric involvement at the time of diagnosis (Tab.1).

Abstract AB1030 Table 1. Clinical and demographic characteristics of patients.

\begin{tabular}{|c|c|c|}
\hline Characteristics & $\begin{array}{l}\text { Patients without NM } \\
\text { of cSLE (173) }\end{array}$ & $\begin{array}{l}\text { Patients with NM } \\
\text { of cSLE (45) }\end{array}$ \\
\hline Girls/boys (girls to boys ratio) & $154 / 19(8.1: 1)$ & $36 / 9(4: 1)$ \\
\hline The average age at onset, years & $12.8 \pm 3.52$ & $13.0 \pm 2.84$ \\
\hline $\begin{array}{l}\text { The median of disease duration at the time of } \\
\text { cSLE verification, months }\end{array}$ & $6.0(2.0 ; 15.0)$ & $5.0(3.0 ; 11.0)$ \\
\hline $\begin{array}{l}\text { The average of disease activity by SLEDAI at } \\
\text { the time of cSLE }\end{array}$ & $12.9 \pm 6.5$ & $22.0 \pm 9.5$ \\
\hline
\end{tabular}

Neurolupus was significantly more frequently detected during the acute development of SLE (46.7\% of patients, $p=0.003)$. Among immunological disorders, patients with NM often had antibodies to RNP ( $p=0.073$ ) in the absence of any differences in other immunological parameters. Of the clinical manifestations in patients with NM, chronic skin lesions were recorded more often $(p=0.076)$, while arthritis was detected relatively less frequently $(p=0.028)$. Serositis and nephritis were diagnosed significantly more often in the group of patients with NM $(p=0.003,0.003$ respectively). Of the hematological disorders, NM was relatively more frequently accompanied by leuco/lymphopenia $(p=0.087)$ and thrombocytopenia $(p=0.077)$. In general, the group of patients with NM was characterized by a higher degree of multiorgan involvment (the number of clinical manifestations was on average 5.6 and 3.7 , respectively, $p$ $<0.0001$ ), but both groups were comparable for number of immunological disorders $(\mathrm{p}=0.49$ ).

As early signs of NM, all patients had mood disorders and impaired school adaptation. In the structure of NM, central nervous system involvement prevailed (89\%). 15 pts $(33.3 \%)$ had experienced more than one NM. Headaches, cognitive dysfunction and cerebrovascular disease were the most common $(28.9 \%, 28.9 \%$ and $24.4 \%$, respectively). Other NM included distal polyneuropathy - $20 \%$, episyndrom - $15.5 \%$, acute confusional state $-11.1 \%$, anxiety disorder $-11.1 \%$, psychosis $-8.9 \%$, paresis $-6.7 \%$, chorea $-4.4 \%$.

Conclusion: Acute onset, multiorgan lesions, mood disorders, impairment of school adaptation in a patient with suspected SLE should prompt investigations into diagnosis of the primary nervous system involvement. Patients with neuropsychiatric disorders require earlier complex aggressive therapy due to greater disease activity and poor prognosis.

Disclosure of Interests: None declared

DOI: 10.1136/annrheumdis-2019-eular.4971 